\title{
Stability Margin Monitoring Method for N Subsystems Cascaded DC Distributed Power Systems
}

\author{
Anshou $\mathrm{LI}^{1,2, a}$, Qi CHEN ${ }^{3, b}$, Jinmin ZHANG $^{4, c}$, Tiecai LI ${ }^{2, d}$, Zicai WANG ${ }^{1}$ \\ ${ }^{1}$ Harbin Institute of Technology, Harbin, 150001, China \\ ${ }^{2}$ Shenzhen Academy of Aerospace Technology, Shenzhen, 518057, China \\ ${ }^{3}$ China Academy of space technology, Beijing, 100094, China \\ ${ }^{4}$ Harbin Institute of Technology Shenzhen Graduate School, Shenzhen, 518055, China \\ aemail: anshouli@aliyun.com, bemail: qw_chen@sina.com, cemail:zjminhit@163.com, \\ demail: litc611@public.hr.hl.cn
}

Keywords: Distributed Power System (DPS); Impedance Criterion; Small Signal Stability; Stability Margin; Stability Margin Monitoring

\begin{abstract}
For two subsystems cascaded DPS, the stability margin monitoring can be implanted via current/voltage perturbation method. But for N subsystems cascaded DPS, the stability margins are different at different interfaces, which results in the stability margin monitoring problem. This paper analyzes the reasons of this phenomenon and proposes a new interface-less stability margin monitoring method. Simulation results verify the proposed method.
\end{abstract}

\section{Introduction}

Distributed power system (DPS) has been widely applied in many areas such as aerospace, aviation, power system, new energy resource, etc., due to its advantages of modularization, integration, extensibility, maintainability and so on. However, with the development of power technology and increasing need, there is an increasing demand in the stability and reliability of DPS. The stability problem of DPS becomes a gradually outstanding problem.

As well as known, DPS with two cascaded subsystems, source subsystem and load subsystem, can be described by equation (1) in small-signal sense [1]:

$$
F=\left.\frac{\hat{u}_{o 2}}{\hat{u}_{i n 1}}\right|_{\hat{i}_{02}=0}=\frac{F_{1} F_{2}}{1+\frac{Z_{o}}{Z_{i n}}}=\frac{F_{1} F_{2}}{1+T_{m}}
$$

where $F_{1}$ and $F_{2}$ are transfer function of source subsystem and load subsystem separately, $T_{m}$ is called minor loop gain, which is the ratio of the output impedance of source subsystem $Z_{o}$, and the input impedance of load subsystem $Z_{i n}$. By treating $T_{m}$ as the open-loop transfer function, Nyquist criterion can be applied to determine the stability of the whole system.

Based on aforementioned theory, many stability criteria [2-5] are proposed to judge the stability of DPS, which can be used to ensure the stability of the DPS in design stage. Besides, online stability margin monitoring are also needed for actual products as the stability margin of DPS may be varied by factors such as parameter variation or changing of operating point. For two subsystems cascaded DPS, current perturbation or voltage perturbation method [7-9], as shown in Fig. 1, is proposed to measure the stability margin. For N subsystems cascaded DPS (as shown in Fig.2), however, there are multiple different interfaces to judge the stability and monitor the stability margin. It could be proved that the stability conclusions are the same [10] at different interfaces. But the stability margins could be quite different at different interfaces, which will be proved in the following part of this paper. Then there is a question that are these stability margin monitoring methods suitable for two subsystems cascaded DPS still suitable for $\mathrm{N}$ subsystems cascaded DPS? This paper tries to answer this question. 


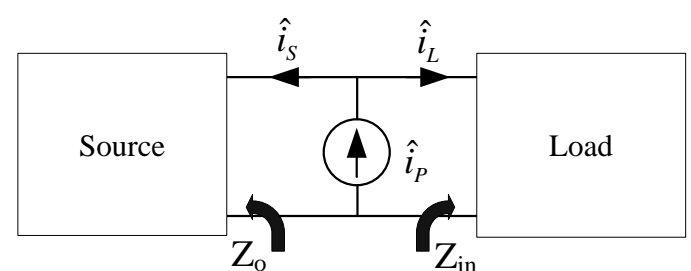

(a) Current perturbation method

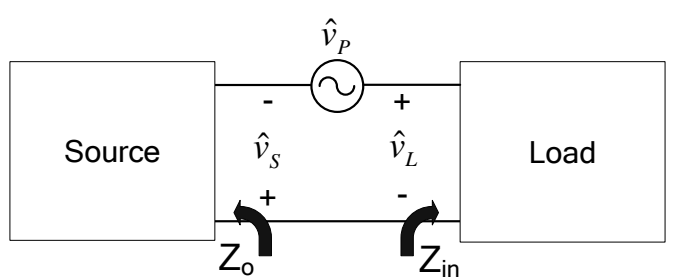

(b) Voltage perturbation method

Fig. 1. Traditional stability margin monitoring method for two subsystems cascaded DPS

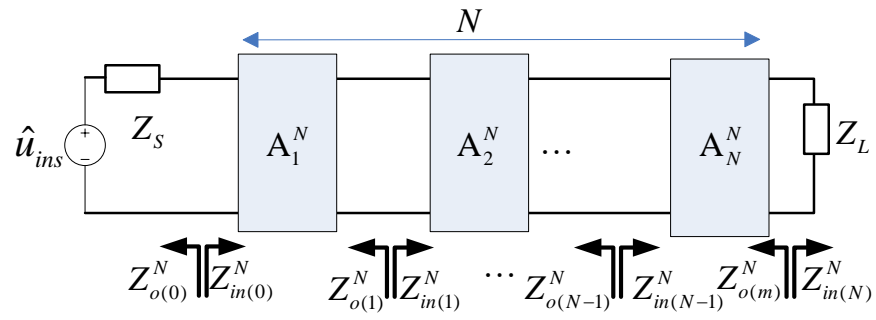

Fig. 2. N subsystems cascaded DPS

\section{The Reasons Why Stability Margins are Different at Different Interfaces}

Take the circuit shown in Fig. 3 as an example, the parameters are as follows: $r=300 \mathrm{~m} \Omega$, $L=10 \mathrm{mH}, \mathrm{C}=40 \mathrm{mF}, \mathrm{R}=24.3 \Omega$, it is easy to know that the system is stable. Fig. 5 is the $T_{m}$ of the system seen from interface A and interface B separately, it can be seen that the $T_{m}$ are different so the stability margin are different too.

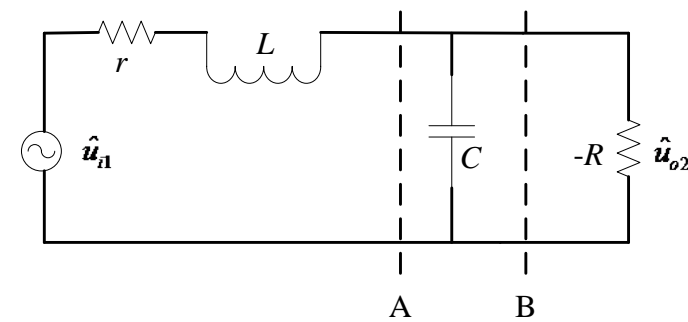

Fig. 3 An example of the small-signal circuit

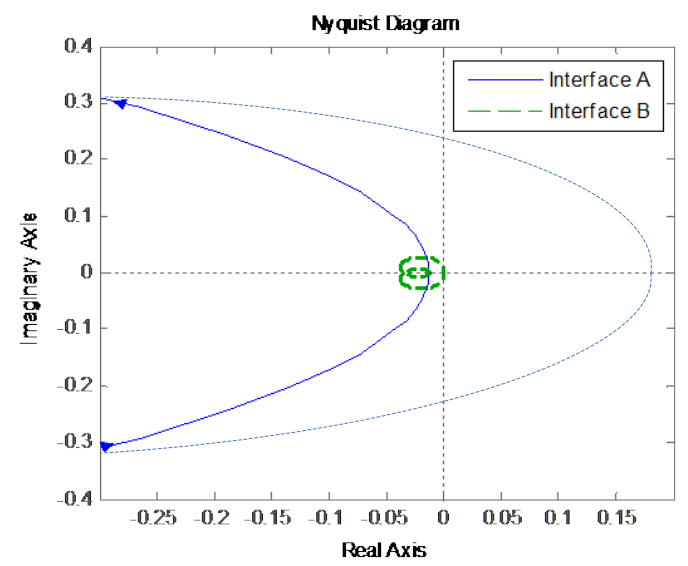

Fig. 4 The Nyquist plot at different interfaces

Why the $T_{m}$ and the stability margin are different seen from different interface? The reason is as follows: assume at interface A, $F=\frac{F_{1} F_{2}}{1+T_{m 1}}$ and at interface B, $F=\frac{F_{3} F_{4}}{1+T_{m 2}}$. Because $F=\frac{F_{1} F_{2}}{1+T_{m 1}}=\frac{F_{3} F_{4}}{1+T_{m 2}}$, and usually $F_{1} F_{2} \neq F_{3} F_{4}$, so $T_{m 1} \neq T_{m 2}$ and the stability margin are different seen from interface A and interface B. Although the roots of $1+T_{m 1}=0$ and $1+T_{m 2}=0$ are the same, the $T_{m 1}$ and $T_{m 2}$ could be very different, which means seen from different interfaces, the stability conclusion are the same but the stability margins could be quite different.

\section{Stability Margin Monitoring Method for N Subsystems Cascaded DPS}

For the same one system, the stability margins are different at different interfaces. This leads to a stability margin monitoring problem: it is unreasonable to use the same impedance forbidden region 
to ensure the stability margin at different interfaces. We should find a new stability margin definition which is interface-less to avoid this problem and a new stability margin monitoring method under the new stability margin definition.

The characteristic equation $1+T_{m}=0$ is used to judge the stability of the whole system $F$ as the stability is only determined by the equation $1+T_{m}=0$. But for stability margin, the situation is different: in traditional way, the $T_{m}$ is taken as open loop transfer function, the stability margin we get is the closed system $\frac{T_{m}}{1+T_{m}}$, not the origin system $F=\frac{F_{1} F_{2}}{1+T_{m}}$. So it is more reasonable to use the index of closed system $F=\frac{F_{1} F_{2}}{1+T_{m}}$ to define the stability margin.

Define the stability margin as :

$$
S=\left|\frac{\frac{\hat{u}_{i n}}{U_{i n}}}{\frac{\hat{u}_{o}}{U_{o}}}\right|_{\hat{i}_{o}=0}=\frac{U_{o}}{U_{i n}} \cdot\left|\frac{\hat{u}_{i n}}{\hat{u}_{o}}\right|_{\hat{i}_{o}=0}
$$

Where $\left|\frac{\hat{u}_{i n}}{U_{i n}}\right|$ means the relative external disturbed input voltage, $\left|\frac{\hat{u}_{o}}{U_{o}}\right|$ means the relative measured disturbed output voltage, $\hat{i}_{o}=0$ means the external disturbed source's current is zero.

When $S \rightarrow 0$, stability margin $\rightarrow 0$; when $S \rightarrow \infty$, stability margin $\rightarrow \infty$. Typically, when $S>0.1=-20 \mathrm{~dB}$, the stability margin of the DPS can be guaranteed in some degree, when $S>10=20 \mathrm{~dB}$, the stability margin of the DPS can be well guaranteed.

Under this stability margin definition, the stability margin $S$ can be obtained by adding an external disturbed voltage source $\hat{u}_{i n}$ in the source side and observe the corresponding disturbed voltage $\hat{u}_{o}$ in the load side as shown in Fig. 5 .

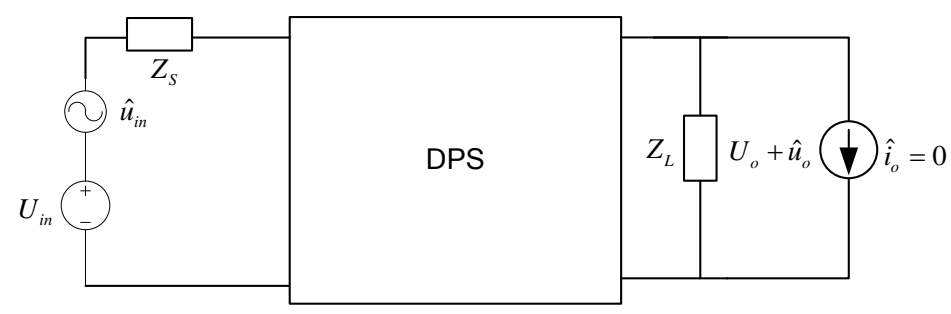

Fig. 5 The new stability margin monitoring method

\section{Simulation results}

As shown in Fig. 6, an example of three buck cascaded DPS is given to verify the proposed stability margin monitoring method. The three converters all work in CCM operating condition. The main parameters of the DPS are also given in Fig. 6. According to the definition of stability margin of equation (2), the stability margin $S$ could be obtained as shown in Fig. 7, it can be seen that the stability margin $S>20 \mathrm{~dB}$, which implies the stability margin is quite good.

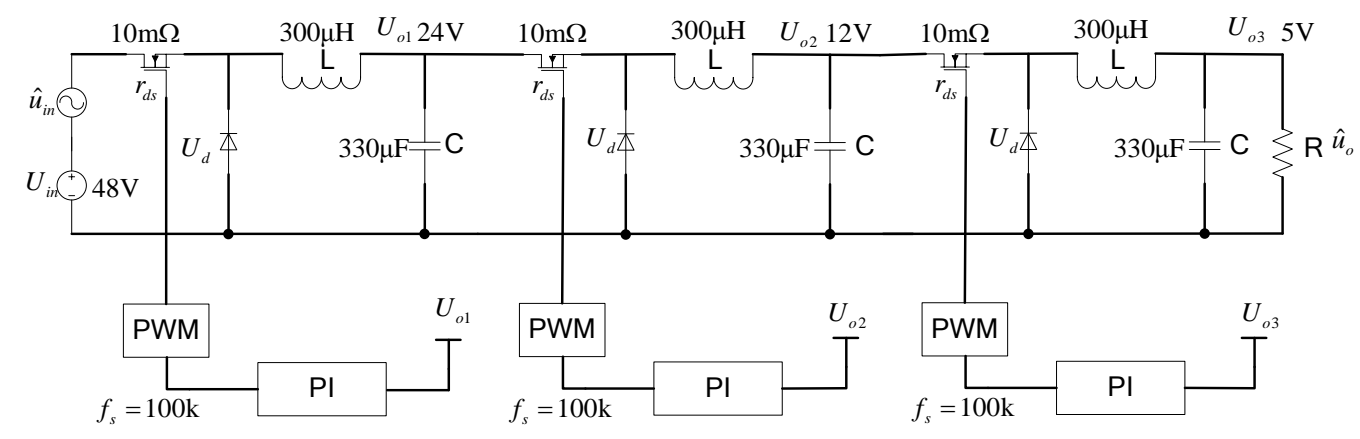

Fig. 6 The three buck cascaded DPS 


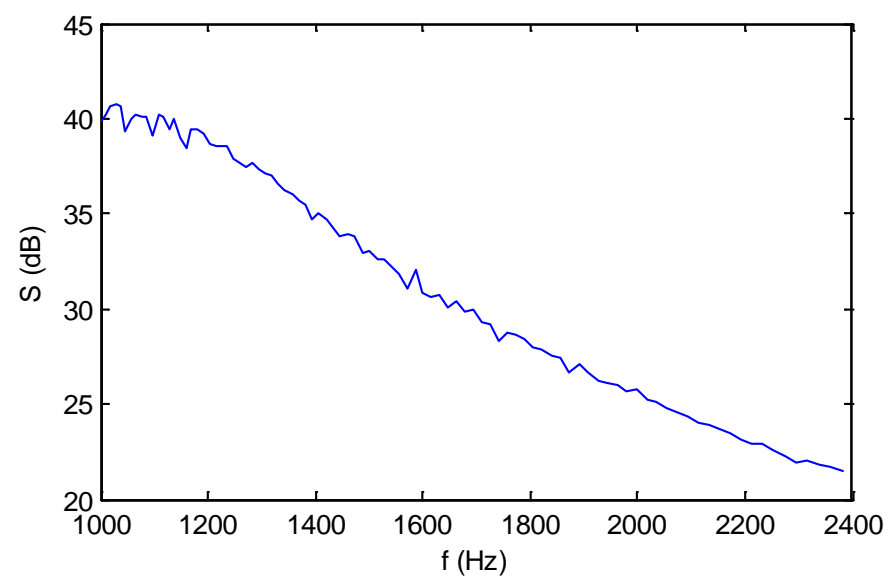

Fig. 7 The stability margin of the three buck cascaded DPS

\section{Conclusion}

For $\mathrm{N}$ subsystems cascaded DPS, the traditional method have different stability margins at different interfaces, which could result in the stability margin monitoring problem. This paper gives the reasons why stability margin are different at different interfaces in theory. To solve this problem, this paper proposes a new stability margin definition and a new stability margin monitoring method. Simulation results verify the proposed method.

\section{References}

[1] Cho B H, Choi B. Analysis and design of multi-stage distributed power system[C]. Proceedings of Telecommunications Energy Conference, 1991:220-226.

[2] Middlebrook R D. Input filter consideration in design and application of switching regulators[C]. IEEE Proceedings of Industrial Application Society Annual Meeting, 1976:94-107.

[3] Wildrick C M, Lee F C, Cho B H, et al. A method of defining the load impedance specification for a Stable Distributed Power System[J]. Power Electronics, 1995, 10(3):280-285.

[4] X Feng, Z Ye, K Xing, et al. Individual load impedance specification for a stable DC distributed power system[C]. IEEE Annual Applied Power Electronics Conference and Exposition (APEC), 1999:923-929.

[5] Sudhoff S D, Glover S F, Lamm P T, et al. Admittance space stability analysis of power electronic systems[J]. IEEE Transaction on Aerospace and Electronic Systems, 2000, 36(3):965-973.

[6] X. Feng, C. Liu, Z. Ye, F C. Lee, D. Borojevic. Monitoring the stability of DC distributed power systems[C]. 1999:367-372.

[7] X. Feng, F C. Lee. On-line measurement on stability margin of DC distributed power system[C]. 2000:1190-1196.

[8] J. Liu, X. Feng, F C. Lee, D. Borojevic. Stability margin monitoring for DC distributed power systems via current/voltage perturbation[C]. 2001:745-751.

[9] J. Liu, X. Feng, F C. Lee, D. Borojevic. Stability margin monitoring for DC distributed power systems via perturbation approaches[J]. Power Electronics, 2003, 18(6):1254-1261

[10] A. Li, D. Zhang, Q. Tong. Impedance stability criterion of complex connected dc distributed power system[C]. International Conference on Mechatronics and Industrial Informatics, ICMII 2013 (IPEMC), 2013:1449-1453. 